\title{
Sintering and Consolidation of Silver Nanoparticles Printed on Polyimide Substrate Films
}

\author{
Sang Hwa Yoon, Jun Ho Lee, Pyoung Chan Lee, and Jae Do Nam* \\ Department of Polymer Science and Engineering, SAINT, Sungkyunkwan University, Suwon 440-746, Korea
}

Hyun-Chul Jung and Yong Soo Oh

Central R\&D Institute, Samsung Electro-Mechanics Co., Ltd., Suwon 443-743, Korea

Tae Sung Kim

School of Mechanical Engineering, Sungkyunkwan University, Suwon 440-746, Korea

Young kwan Lee

Department of Polymer Chemical Engineering, Sungkyunkwan University, Suwon 440-746, Korea

Received July 12, 2008; Revised February 16, 2009; Accepted February 17, 2009

\begin{abstract}
We investigated the sintering and consolidation phenomena of silver nanoparticles under various thermal treatment conditions when they were patterned by a contact printing technique on polyimide substrate films. The sintering of metastable silver nanoparticles commenced at $180{ }^{\circ} \mathrm{C}$, where the point necks were formed at the contact points of the nanoparticles to reduce the overall surface area and the overall surface energy. As the temperature was increased up to $250^{\circ} \mathrm{C}$, silver atoms diffused from the grain boundaries at the intersections and continued to deposit on the interior surface of the pores, thereby filling up the remaining space. When the consolidation temperature exceeded $270{ }^{\circ} \mathrm{C}$, the capillary force between the spherical silver particles and polyimide flat surface induced the permanent deformation of the polyimide films, leaving crater-shaped indentation marks. The bonding force between the patterned silver metal and polyimide substrate was greatly increased by the heat treatment temperature and the mechanical interlocking by the metal particle indentation.
\end{abstract}

Keywords: silver nanoparticles, sintering, polyimide, capillary force.

\section{Introduction}

Compared with the conventional photolithography/etching processes, the direct printing of metal particle pastes has mainly been used for electronics patterning, because it is environmentally friendly in terms of the reduction in the amount of material loss and number of processing steps, thus allowing for high productivity and mass customization. ${ }^{1-6}$ Direct printing techniques including the ink-jet and microcontact printing processes can draw conductive lines directly patterned on the substrate in one step using silver, gold, or other nanoparticles. ${ }^{7-10}$ In particular, if nanoparticles are used for direct printing, the conductive lines can be solidified at substantially decreased temperatures due to the extremely high surface free energy of nano-sized metal particles. $^{11,12}$ When the patterned nanoparticles are heated, sin-

*Corresponding Author. E-mail: jdnam@skku.edu tering occurs below the melting temperature of the nanoparticles accompanied with consolidation and densification of the patterned parts. Accordingly, the sintering and consolidation processing nano-sized particles may well different from the micrometer-sized particles, which should be understood especially for electronic device structuring and design.

In the direct printing process, metal nanoparticles can be printed on various substrates for use in electronic and electric device fabrication. ${ }^{13-20}$ Among the possible polymer substrates, polyimide is one of the most widely used materials in the field of opto-electronic devices such as electric insulators, circuit boards, waveguides, and alignment layers of liquid-crystal displays, due to its excellent thermal and mechanical stabilities, e.g., tensile modulus of $2.5 \mathrm{GPa}$, volume DC resistivity of the order of $10^{18} \Omega \mathrm{m}$, low thermal conductivity, and high glass transition temperature of over $400{ }^{\circ} \mathrm{C} .{ }^{21-23}$ It is also expected that the direct printing of metal nanoparticles on flexible polyimide substrate films 
can be used in such applications as flexible microelectromechanical (MEMS), semiconductor devices, flexible displays, etc,. However, it has been pointed out that nanoparticle immobilization on a polyimide substrate is difficult, due to the poor adhesion characteristics of polyimide to other materials. ${ }^{24-26}$ Accordingly, the polyimide surface has been modified by various methods to improve its adhesion characteristics with metals, including wet chemical treatments, ${ }^{27-31}$ plasma treatment, $^{32-35}$ laser irradiation ${ }^{36,37}$ and ion beam treatment. ${ }^{21}$

Herein, we investigated the sintering and consolidation of silver nanoparticles on polyimide substrate films subjected to thermal treatment around the melting temperature of the silver nanoparticles. The different stages of sintering and consolidation of the silver nanoparticles in the vicinity of the polyimide surface were investigated. We also investigated the interface morphology between the silver nanoparticles and the polyimide surface during the sintering procedure.

\section{Experimental}

The silver nanoparticles used in this study are shown in Figure 1. The size of the particles dispersed in diethylene glycol (DEG) as a cosolvent to water at a ratio of $50 \mathrm{wt} \%$ was ca. $50 \mathrm{~nm}$. The polymer substrate investigated in this study was a commercially-available Kapton film (HN, Dupont). The silver nanoparticle paste was patterned on the polyimide films using the microcontact printing technique. The master and elastomeric polymer stamp used for contact printing was fabricated as reported previously. ${ }^{38}$ First, the polymer stamp was slightly inked with the silver nanoparticles. Then, it was placed in contact with the polyimide substrate and held there under slight pressure for $1 \mathrm{~min}$ at room temperature. The patterned substrate was dried at room temperature for $30 \mathrm{~min}$ and then heated up to various temperatures between 180 and $300{ }^{\circ} \mathrm{C}$.

Differential scanning calorimetry (DSC) was used to study the thermal properties of the silver nanoparticles using a TA Instruments DSC 910 in a nitrogen atmosphere at a heating rate of $10{ }^{\circ} \mathrm{C} / \mathrm{min}$. The sintered nanoparticles were investigated by a scanning electron microscopy (JSM7000F, JEOL Tokyo, Japan) and optical microscopy (Model: I Camscope G, Plus win, Korea). The adhesion between the silver pattern and polyimide substrate was measured by the scotch tape test using a $3 \mathrm{M}$ Scotch Brand Tape (\#3750-G). The tape was slowly $(\sim 2 \mathrm{~cm} / \mathrm{s})$ peeled off by hand from the polyimide substrate at the angle of $160^{\circ} \sim 170^{\circ}$.

\section{Results and Discussion}

As seen in Figure 1, the DSC thermogram shows that the melting point of the silver nanoparticles is $258{ }^{\circ} \mathrm{C}$, which should be compared with the value of $962^{\circ} \mathrm{C}$ for bulk silver metal. This low melting temperature of the silver nanoparticles is due to the relatively large ratio of surface atoms to inner atoms. ${ }^{39}$ The high proportion of surface atoms drastically decreases the melting temperature, because the surface energy of the unbound surface atoms is extremely high. The melting point of nanoparticles has been inversely correlated with their radius, as follows: ${ }^{40}$

$$
T_{n}=T_{b}-\frac{2 V_{m, l}(l) \gamma_{s l} T_{b}}{\Delta H_{m} r}
$$

where $T_{n}$ is the melting point of nanoparticles with radius $r$, $T_{b}$ is the melting point of the bulk material, $V_{m, l}$ is the molar volume of the liquid, $\gamma_{s l}$ is the interfacial tension between the solid and the liquid surface layer, and $\Delta H_{m}$ is the bulk latent heat of melting. According to this equation, when the particle diameter is decreased, its second term is radically increased, resulting in a substantial decrease in the melting temperature of the nanoparticles. For example, the melting point of gold nanoparticles whose size is of the order of a few nanometers is in the range of $300-400{ }^{\circ} \mathrm{C}$, which should be compared with $1,064{ }^{\circ} \mathrm{C}$ for the bulk form of gold..$^{39}$
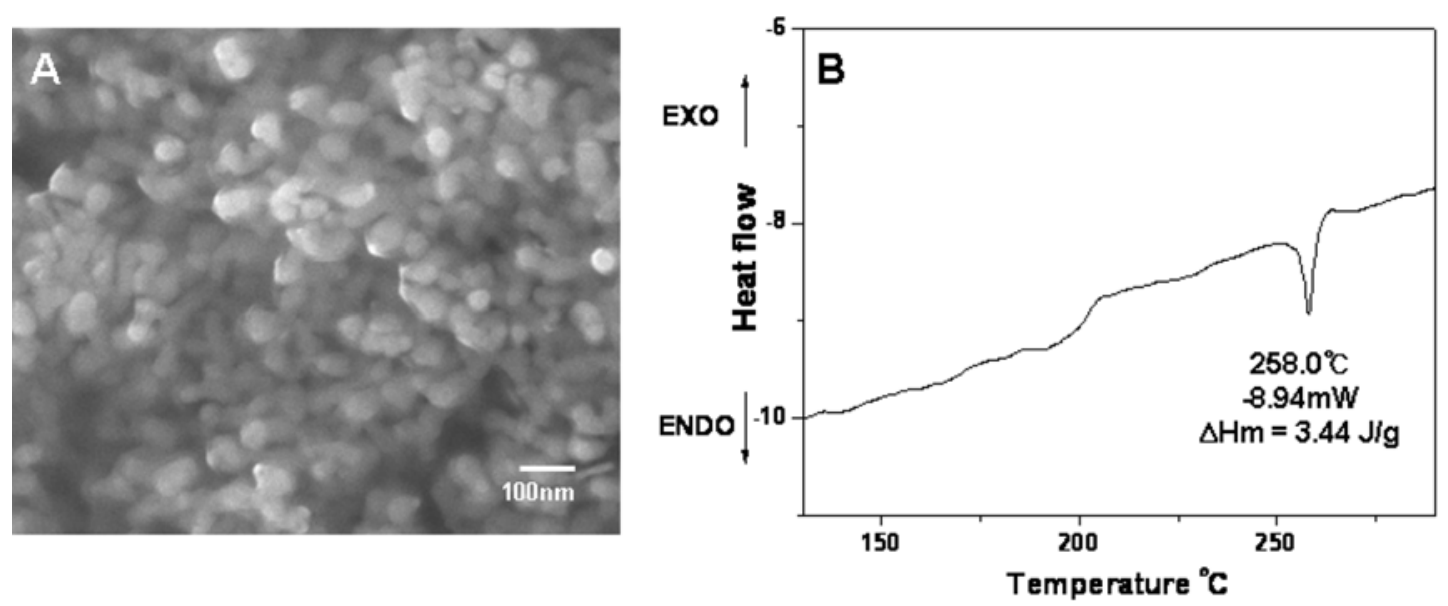

Figure 1. SEM images (A) and DSC curve (B) of silver nanoparticles used in this study. 
Figure 2 shows the optical micrographic pictures of the silver patterns formed by the microcontact printing on the polyimide films. The light color areas of the image are the silver nanoparticles patterns. The silver patterns exhibit sharp edges and the pattern dimensions are in good agreement with the line-to-space distance specifications of the polymer stamp of $150 \mu \mathrm{m} / 90 \mu \mathrm{m}$. The silver nanoparticles are only found where the polymer stamp was in contact with the polyimide substrate and the generated silver layer is crack free.

As schematically shown in Figure 3 sintering is normally thought to occur in four stages. ${ }^{41}$ When the particles are first placed in contact each other, instantaneous neck formation takes place. Once it is formed, the neck grows rapidly by several different mechanisms including surface diffusion, lattice diffusion from the surface, vapor transport, grain boundary diffusion, and lattice diffusion from the grain boundary accompanied by plastic flow (Figure 3(B)). This
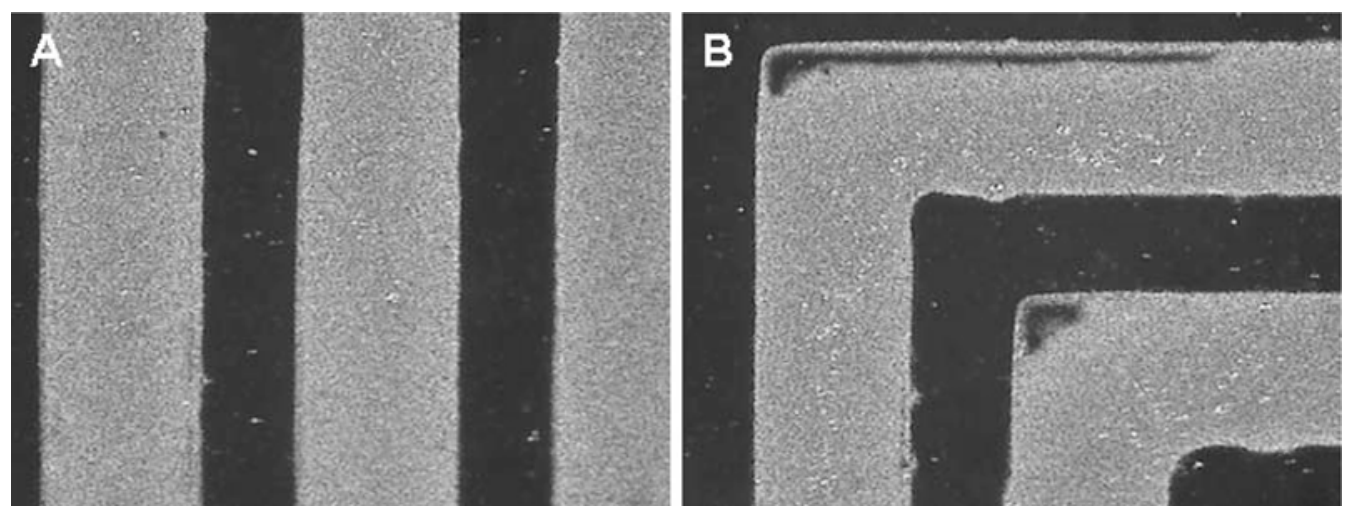

Figure 2. Optical micrographs of silver nanoparticle patterns formed on polyimide substrate film.
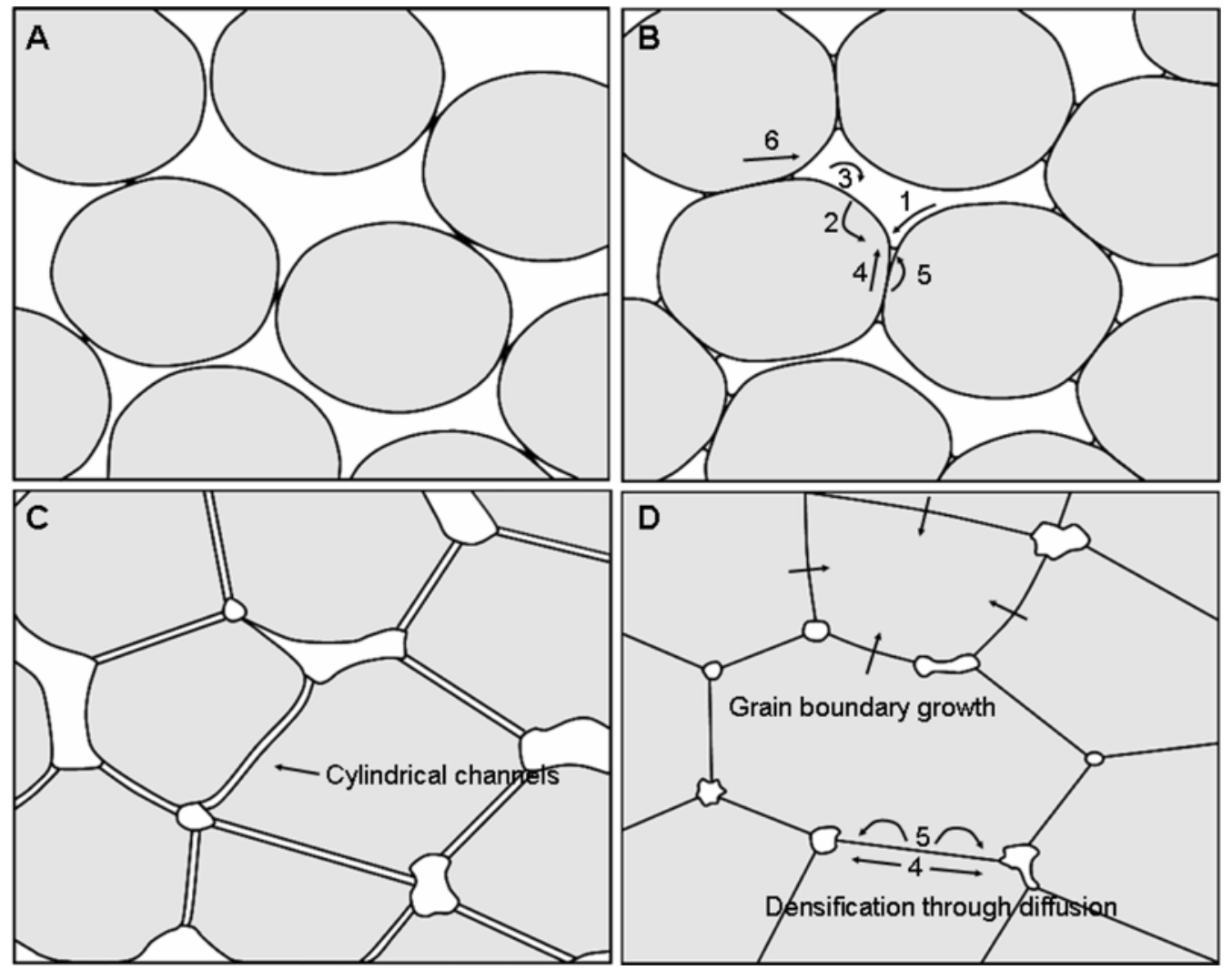

Figure 3. Model structure of the sintering process exhibiting (A) instantaneous neck formation, (B) neck growth, (C) cylindrical channels at boundaries and (D) pore elimination. The transport phenomena designated by the numbers in (B) and (D) represent surface diffusion (1), lattice diffusion from the surface (2), vapor transport (3), grain boundary diffusion (4), lattice diffusion from the grain boundary (5) and plastic flow (6). 
stage is considered to last until the radius of the neck between the particles reaches $0.4-0.5$ of the particle radius, at which point the initial density of $0.5-0.6$ of the theoretical density increases to around 0.65 with a linear shrinkage of $3-5 \%{ }^{41}$ For nanoparticles, it should be mentioned that the sintering mechanism may be significantly different from the traditional one, due to their high surface curvature and unbound surface atoms, which give them an extremely high surface free energy. In nanoparticle sintering, it was reported that the surface and the grain boundary diffusion were the most significant transport processes in the sintering process of $\mathrm{Cu}$ and $\mathrm{Ag}$ nanoparticles. ${ }^{42}$

The intermediate stage (Figure 3(C)) begins when the pores reach their equilibrium shapes, as dictated by the surface and interfacial tensions. In this stage, the pore phase is continuous and gives cylindrical channels of porosity sitting along the grain edges, as illustrated in Figure 3(C). Consoli- dation is considered to occur by the reduction of the pore volume in this stage. The final stage of sintering begins when the pores pinch off and become isolated at the grain corners (Figure 3(D)), at which point they may shrink continuously with time and disappear.

Figure 4 shows the SEM images of the silver nanoparticles sintered at different temperatures on the surface of the polyimide films. For these micrographs, the sintered samples were microtomed by a diamond saw in order to observe the cross-sectioned image of the voids and nanoparticles. When the nanoparticles are heated up to $180{ }^{\circ} \mathrm{C}$ (Figures 4(A) and (B)), necks are formed so as to interconnect the nanoparticles and leaving a large amount of pores, which seemingly corresponds to the schematic representation in Figure 4(B). In this stage, it is believed that the silver atoms diffuse from the nanoparticles at the intersections between the differently oriented particles and continue to deposit on
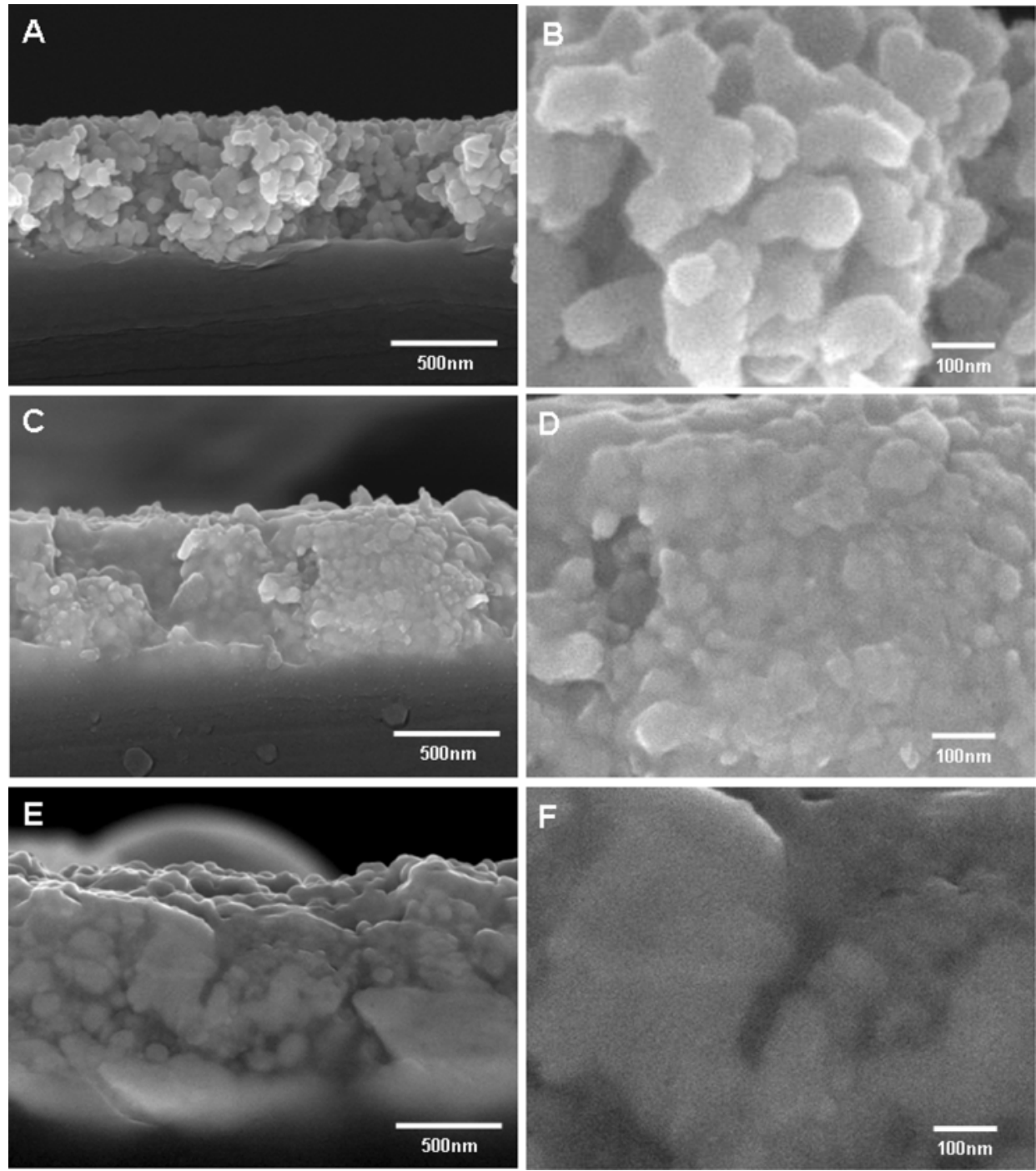

Figure 4. SEM images of silver nanoparticles coated on polyimide film after the heat treatment at $180{ }^{\circ} \mathrm{C}(\mathrm{A}, \mathrm{B}), 250{ }^{\circ} \mathrm{C}(\mathrm{C}, \mathrm{D})$ and $290{ }^{\circ} \mathrm{C}(\mathrm{E}, \mathrm{F})$. 


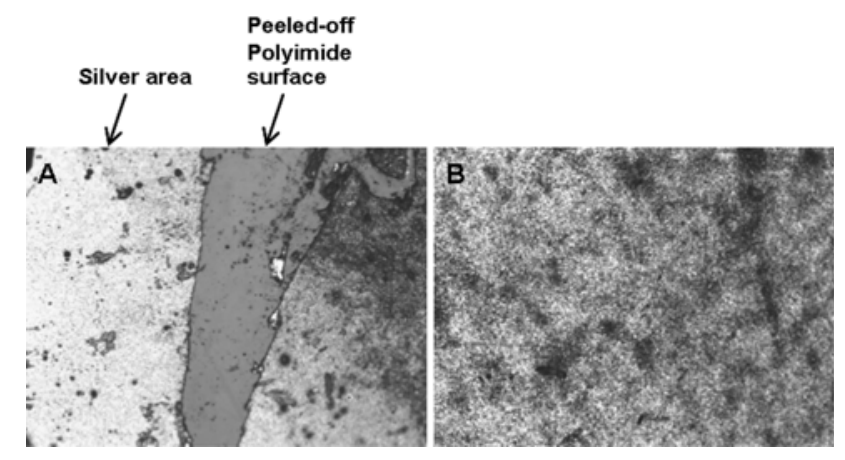

Figure 5. Optical micrographs of polyimide film surface after the scotch tape test. For the specimens heat treated at (A) $180{ }^{\circ} \mathrm{C}$ and (B) $300{ }^{\circ} \mathrm{C}$.

the neck region.

Upon further heating, the neck areas increase in size and the pores are isolated to form a cylindrical shape, which corresponds to the characteristic feature of the intermediate stage of sintering in Figure 4(C). As the temperature is increased up to $250{ }^{\circ} \mathrm{C}$, as shown in Figures $4(\mathrm{C})$ and (D), the pores are significantly decreased in size and become more isolated. Herein, it is difficult to distinguish the particle shape and the neck regions, which indicates that the sintering enters the final stage. Upon further heating above $290{ }^{\circ} \mathrm{C}$, grain growth continues to occur to form dense and porous polycrystalline silver (Figures $4(\mathrm{E})$ and $(\mathrm{F})$ ). As can be seen, there are no pores remaining in the final stage of sintering. In this final stage, the silver atoms move from the convex surface on one side of the grain boundary to the con- cave surface on the other side, due to chemical potential differences, to give a homogeneous phase of sintered materials.

Figure 5 shows the silver nanoparticles sintered at different temperatures after the scotch tape test. The peeled-off silver nanoparticles after sintering at $180{ }^{\circ} \mathrm{C}$ are shown in Figure 5(A). It can be seen that the silver nanoparticles are partially detached from the polyimide film, due to their poor mechanical adhesion at the metal/polymer interface. However, the silver nanoparticles are not detached by the scotch tape when they are sintered at $300{ }^{\circ} \mathrm{C}$ (Figure 5(B)), demonstrating that the bond strength of the nanoparticles to the polymer surface is high enough to sustain the peel-off stress in this stage.

Figure 6 shows the polyimide film surface after the sintered silver layer was peeled off by means of a scotch tape. The peeled-off polyimide surface after the sintering of the silver pattern at $180{ }^{\circ} \mathrm{C}$ is smooth and maintains the pristine state of the film (Figure 6(A)). As the temperature is increased up to $250{ }^{\circ} \mathrm{C}$, there are some meniscus-shaped marks left on the polyimide surface, as shown in Figure 6(B). At $270{ }^{\circ} \mathrm{C}$, the depth of the meniscus patterns is increased significantly and some remaining silver nanoparticles can be seen in Figure $6(\mathrm{C})$. When the sintering temperature is over $290{ }^{\circ} \mathrm{C}$, the polyimide surface is significantly deformed to produce particle shapes (Figure 6(D)).

The capillary force between the spherical silver nanoparticles and polyimide flat surface may induce plastic deformation of the polyimide films when they change into the rubbery state during heating. As seen in Figure 7, the driving force of this surface deformation of the polyimide film
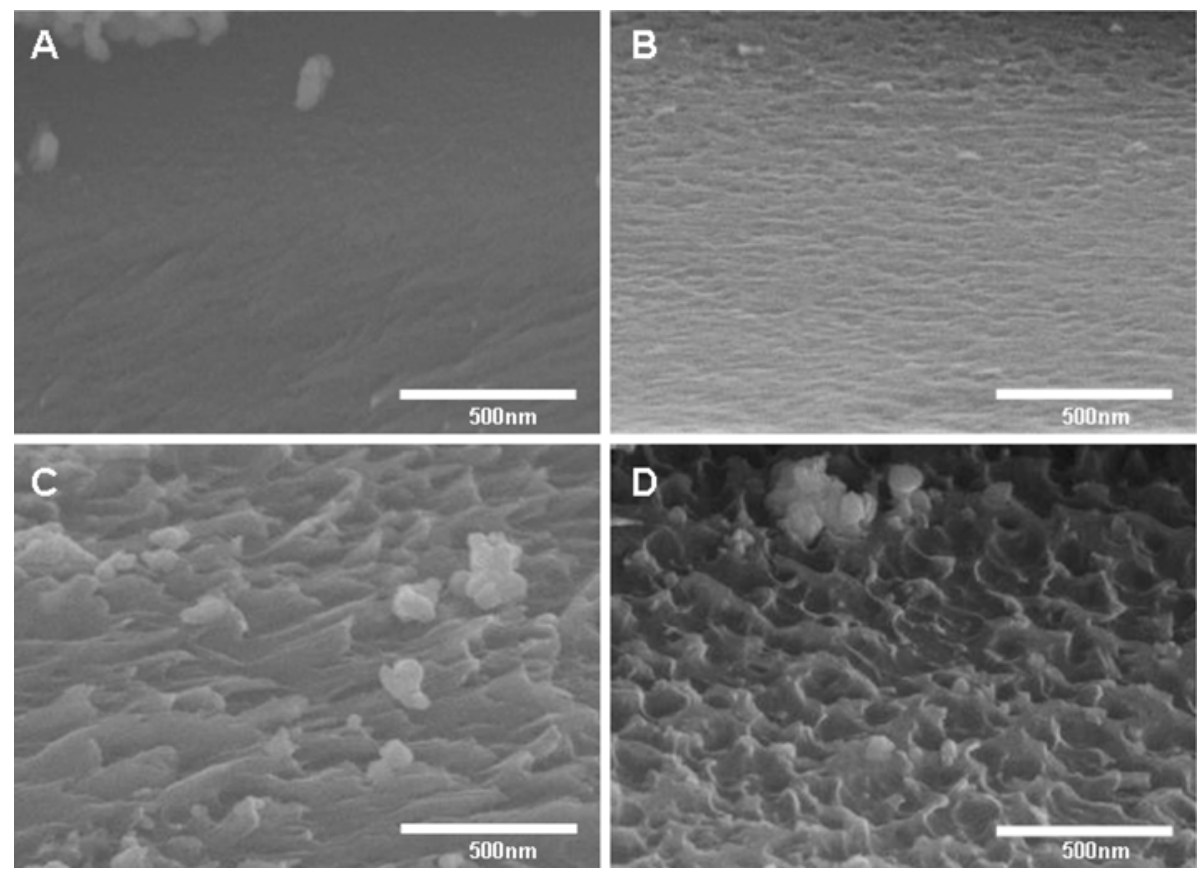

Figure 6. SEM images of polyimide film surface after the silver layer was peeled off. The heat treatment temperatures are (A) $180{ }^{\circ} \mathrm{C}$, (B) $250{ }^{\circ} \mathrm{C},(\mathrm{C}) 270{ }^{\circ} \mathrm{C}$ and (D) $290^{\circ} \mathrm{C}$. 
A

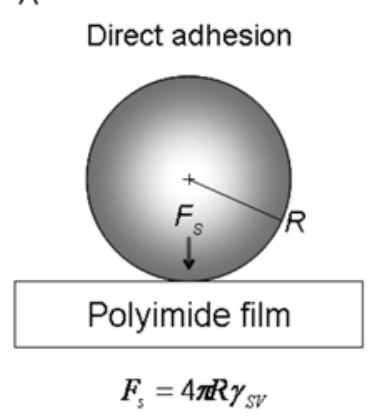

B

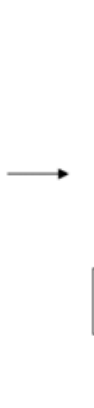

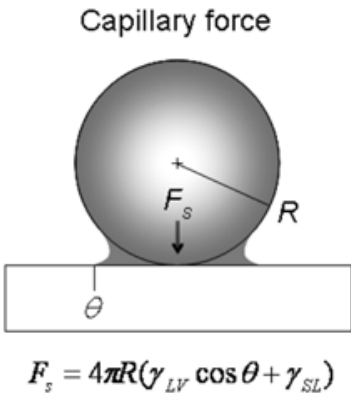

C Surface deformation

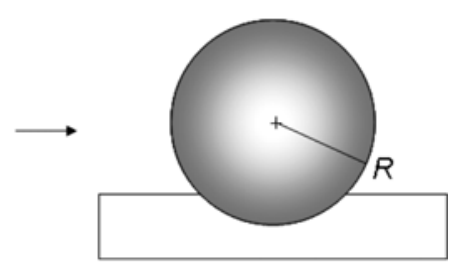

Figure 7. Schematic presentations for surface deformation of silver nanoparticles on polyimide substrate film surface.

is a combination of the adhesion and the capillary forces between the silver nanoparticles and polyimide (Figure 7). Below the melting temperature of the silver nanoparticles, there is only the direct adhesion force between the two contacting solids, represented by $F_{S}=4 \pi R \gamma_{S V}$, where $F_{S}$ is the adhesion force, $R$ is the radius of the nanoparticles and $\gamma_{S V}$ is the interfacial tension between the solid and vapor. When the temperature is increased to around the melting temperature of the silver nanoparticles, the Laplace pressure of the curved menisci is developed between the silver nanoparticles and the polyimide flat surface, as follows: $F_{S}=4 \pi R\left(\gamma_{L V}\right.$ $\left.\cos \theta+\gamma_{S L}\right)$ where $\gamma_{L V}$ is the interfacial tension between the liquid and vapor, $\theta$ is the contact angle between the nanoparticles and substrate and $\gamma_{S L}$ is the interfacial tension between the solid and vapor. As this force develops and the increased temperature causes the modulus of the polyimide to decrease, the nanoparticles may be forced to sink down under the polyimide surface. We believe that the surface deformation of the polyimide film induced at elevated temperatures substantially increases the metal-polymer adhesion force through mechanical interlocking.

\section{Conclusions}

The sintering of $50 \mathrm{~nm}$ sized silver nanoparticles was investigated in the temperature range of $180 \sim 290{ }^{\circ} \mathrm{C}$ around the melting temperature of nanoprarticles. At around $180{ }^{\circ} \mathrm{C}$, necks formed at the contact points of the nanoparticles to reduce the surface energy. As the temperature was increased up to $250{ }^{\circ} \mathrm{C}$, silver atoms from the grain boundary filled up the pores and remaining space. The neck region and pores almost disappeared due to plastic flow at $270{ }^{\circ} \mathrm{C}$. Due to the capillary force between the Ag particles and PI surface, the PI surface started deforming, leading to the formation of dimples. Above $290{ }^{\circ} \mathrm{C}$, the grain boundaries between the particles disappeared and the size of the particles increased significantly. Because of the capillary force between the spherical silver particles and polyimide flat surface, the polyimide surface deformed elastically in the rubbery state.
Acknowledgements. This work was supported by Gyeonggi Province through the Gyeonggi Regional Research Center (GRRC) Program in Sungkyunkwan University.

\section{References}

(1) B. J. D. Gans, P. C. Duineveld, and U. S. Schubert, Adv. Mater., 16, 203 (2004).

(2) R. F. Service, Science, 304, 675 (2004).

(3) C. W. Sele, T. V. Werne, R. H. Friend, and H. Sirringhaus, Adv. Mater., 17, 997 (2005).

(4) M. S. Park, T. H. Lee, Y. M. Jeon, J. G. Kim, and M. S. Gong, Macromol. Res., 16, 308 (2008).

(5) J. K. Kim and H. Ahn, Macromol. Res., 16, 163 (2008).

(6) G. P. Kim, Y. S. Jung, S. B. Yoon, D. W. Kim, and S. H. Baeck, Macromol. Res., 15, 693 (2007).

(7) S. Magdassi, A. Bassa, Y. Vinetsky, and A. Kamyshny, Chem. Mater, 15, 2208 (2003).

(8) A. Kamyshny, M. Ben-Moshe, S. Aviezer, and S. Magdassi, Macromol. Rapid Commun., 26, 281 (2005).

(9) Y. Li, Y. Wu, and B. S. Ong, J. Am. Chem. Soc., 127, 3266 (2005).

(10) D. Huang, F. Liao, S. Molesa, D. Redinger, and V. Subramanian, J. Electrochem. Soc., 150, 412 (2003).

(11) M. C. Daniel and D. Austruc, Chem. Rev., 104, 293 (2004).

(12) P. V. Kamat, J. Phys. Chem. B, 106, 7729 (2002).

(13) G. Chumanov, K. Sokolov, B. Gregory, and T. M. J. Cotton, Phys. Chem., 99, 9466 (1995).

(14) K. C. Grabar, R. G. Freeman, M. B. Hommer, and M. J. Natan, Anal.Chem., 67, 735 (1995).

(15) G. Chumanov, K. Sokolov, and T. M. J. Cotton, Phys. Chem., 100, 5166 (1996).

(16) S. Malynych, I. Luzinov, and G. Chumanov, Phys. Chem. B, 106, 1280 (2002).

(17) L. Xu, J. Liao, L. Huang, D. Ou, Z. Guo, H. Zhang, and C. Ge, Thin Solid Films, 434, 121 (2003).

(18) Y. Masuda, T. Koumura, T. Okawa, and K. Koumoto, J. Colloid Interf. Sci., 263, 190 (2003).

(19) K. Bandyopadhyay, V. Patil, K. Vijayamohanan, and M. Sastry, Langmuir, 13, 5244 (1997).

(20) Z. Zhong, B. Gates, Y. Xia, and D. Qin, Langmuir, 16, 10369 (2000). 
(21) M. K. Ghosh and K. L. Mittal, Polyimides: Fundamentals and Applications, Marcel Dekker, New York, 1996, pp 121.

(22) D. Wilson, H. D. Stenzenberger, and P. M. Hergenrother, Polyimides, Chapman and Hall, New York, 1990, pp 370.

(23) Available from <http://dupont.com/kapton/general/H-384922.pdf $>$.

(24) D. Y. Shih, N. Klymko, R. Flitsch, J. Paraszczak, and S. Nunes, J. Vac. Sci. Technol. A, 9, 2704 (1991).

(25) D. L. Pappas and J. J. Cuomo, J. Vac. Sci. Technol. A, 9, 2704 (1991).

(26) T. Strunskus, M. Grunze, G. Kochendoerfer, and C. Woll, Langmuir, 12, 2712 (1996).

(27) K. W. Lee, S. P. Kowalczyk, and J. M. Shaw, Macromolecules, 23, 2097 (1990).

(28) K. W. Lee, S. P. Kowalczyk, and J. M. Shaw, Langmuir, 7, 2450 (1991).

(29) K. W. Lee and A. Viehbeck, IBM J. Res. Dev., 38, 457 (1994).

(30) I. Ghosh, J. Konar, and A. K. Bhowmick, J. Adhes. Sci. Technol., 11, 877 (1997).

(31) R. R. Thomas, S. L. Buchwalter, L. P. Buchwalter, and T. H.
Chao, Macromolecules, 25, 4559 (1992).

(32) G. Rozovskis, J. Vinkevicius, and J. Jaciauskiene, J. Adhes. Sci. Technol., 10, 399 (1996).

(33) N. Inagaki, S. Tasaka, H. Ohmori, and S. Mibu, J. Adhes. Sci. Technol., 10, 243 (1996).

(34) H. K. Yun, K. Cho, J. K. Kim, C. E. Park, S. M. Sim, S. Y. Oh, and J. M. Park, J. Adhes. Sci. Technol., 11, 95 (1997).

(35) M. Strobel, C. Lyons, and K. L. Mittal, Plasma Surface Modification of Polymers, VSP Publications, Utrecht, 1994, pp 201.

(36) H. Hiraoka and S. Lazare, Appl. Surf. Sci., 46, 264 (1990).

(37) H. Niino and A. Yabe, Appl. Surf. Sci., 69, 1 (1996).

(38) A. Kumar, H. A. Biebuyck, and G. M. Whitesides, Langmuir, 19, 1498 (1994).

(39) P. Buffat and J. P. Borel, Phys. Rev. A, 13, 2287 (1975).

(40) W. Thomson, Philos. Mag., 42, 448 (1871).

(41) M. N. Rahaman, Ceramic processing and sintering, Dekker, New York, 1969, p. 389.

(42) P. Zeng, S. Zajac, and P. C. Clapp, Mater. Sci. Eng. A, 252, 301 (1998). 